\title{
Performance Study of Dymo on Demand Protocol using Qualnet 5.0 Simulator
}

\author{
${ }^{1}$ Mrs. C.Chandralekha, ${ }^{2}$ Balachandar. R, \\ ${ }^{I}$ Associate Professor, Dept. of MCA, SNS College of Technology, Coimbatore. \\ ${ }^{2}$ III MCA, SNS College of Technology, Coimbatore.
}

\begin{abstract}
Wireless adhoc network support rapid on demand and adaptive communication among the nodes due to their self configurable and autonomous nature and lack of fixed infrastructure. Since adhoc network rely on the collaboration principle the issue of key distribution and effective group by management in such networks represents two of the most important problem. This paper explore in depth the requirements of this set of applications scenarios and purposes from machine learning based on demand routing paradign.Its primary benefits are flexibility to optimize routing over a variety of property such as route length easy recovery after node failure and native support for sink mobility. As efficient approach to its consider routing algorithm in which network connectivity is determined in the process of establishing routes. The shortest path from source to designation in a static network is optimal route this idea is not easily extended to Manet.Factors such as power expended, variable wireless link quality, propagation, Pathless, fading, multiuser interference and topological changes become relevant use. Performance is an interesting issue for different protocols. Routing In adhoc networks is challenging due to mobility of nodes. Dynamic adhoc on demand routing protocols is one in which is intended for the use by mobile nodes in wireless adhoc multihop adhoc network. It can adopt changing network topology and determine unicast routes between nodes within the network. Mobile Adhoc network has evolved fast in the last 10 year into standard in the communication world. Manet is self configuring network of mobile node connected by wireless links to form an arbitrary topology without the use of existing infrastructure. In this paper we deal with new implementation of routing protocol dynamic manet on demand, It offer adaptation to charge the network topology and determine unicast router between nodes within the network on demand. Our goal is to carry out a systematic performance study of on demand dynamic routing protocols (DYMO) which initiate routing activities on demand basis. High routing load .Performance analyzed using network simulator qualnet 5.0. It mobility network size and load is analyzed. We make recommendation about how the performance how to improved.
\end{abstract}

Keywords: - Adhoc networks. On demand network, ADOV, DYMO, DSR, QUALNET1.

\section{INTRODUCTION:}

Manets were traditionally developed for networks; A manet is a dynamic Wireless network wireless network with or without fixed infrastructure. Nodes may move freely and organize themselves, arbitrarily thus the network topology may charge rapidly and unpredictably .Each node may communicate directly with any node. Communication beyond that range is achieved by using intermediate nodes to relay messages hop by hop such a route may include multiple hops and such a route may include multiple hops and therefore routing network may be a multihop network. Manet donot depend on centralized administration rather than each node acts as an independent router and typically also as an application node generating and receiving application data. The routing protocols have unique protocols with their unique characteristics. .Hence in order to find out the most adaptive and efficient routing protocol for the highly dynamic topology in Adhocnetwork, the routing protocols behavior has to be analyzed and using varied node mobility, speed traffic and network size.

\section{MOBILE ADHOC NETWORKING PROTOCOLS:}

The main problem with adhoc network is how to send a message from one node to another with no direct link. The nodes are moving around unpredictable and it is very challenging, which nodes that are directly linked together, The Topology of an Adhoc network is constantly changing and it is very difficult for routing process. Manet doesn't depend on centralized administration rather each node acts as an independent router and typically also an application node. Routing protocols can be divided into the following categories

1. Unicast routing protocols

2. Topology based routing protocol

○ Proactive routing

$\circ$ Reactive routing

○ Hybrid routing

3. Multicast routing protocol 


\section{Broadcast routing algorithm}

Manet routing protocols can be classified into two main categories proactive routing can be classified into two main categories proactive routing protocol attempt to maintain routing information .For every pair of network nodes by actively propagating route updates. Reactive protocols establish a route to a destination only when needed .The source node imitate a router discovery process when the root is required and once a router has been established .It is maintained until each destination only when needed .The source node initiates a router discovery process when the root is required and once a router has been established. It is maintained until each destination becomes accessible or until the router is no longer used.

As reactive routing protocol flood the network to discover the route, they are not optimal in terms of bandwidth utilization but they scale well in the frequency of topology change. it can Be classified into the categories source routing and hop by hop routing. Source routing: in source routing on demand protocols each data packets carry complete source to destination address. Each intermediate node forwards these packets according to the information kept in the header of each packet so the intermediate nodes does not need up to date routing information. For each active route in order to forward the packet towards the destination .

\section{HOP BY HOP}

Each data occur by coding routing request packets through packets only carry the destination address and next hop address so that each intermediate node in path to destination uses its routing table to forward each data packet towards the destination uses its routing table to forward each data packet towards the destination, There are three on demand routing protocols

1. Adhoc on demand routing

2. Dynamic source routing

3. Dynamic Manet on demand routing protocol

\section{ON DEMAND ROUTING PROTOCOLS}

These protocols take a lazy approach to routing .When a source want to send to a designation, It invokes the route discovery mechanisms to find the path to the desination. The route remains valid till the destination is reachable or until the route is no longer needed. Unlike, Table driven, reactive or on demand routing protocols do not main an updated table. An important feature of AODV is the maintenance of timer based states in each node, regarding utilization of individual routing table entries. A routing table is expired if not used recently

A set of predecessor is maintained for each routing Table entry. The benefits of AODV are that it favours the least congested route instead of the shortest route and it supports path unicast and multicast packet transmission even for the nodes in constant movement. AODV does not put any additional overhead on data packets as it does not make use of source routing so valid is expired and the determination of a reasonable expiry time is difficult .When network grows performance metrics began to decrease..TORA is an adaptive and efficient routing algorithm primarily designed for mobile adhoc Networks. Main features are propagated to control message only around the points of failures when a link failure occurs. TORA is scale up a large networks but has higher overhead to smaller Network Routes are adopted according to topological change Route is erased on reception of CLR packets. TORA faces a severe security problem. AODV can gather only limited amount of routing information.

DSR and AODV are the only two competing reactive routing protocols Both of them consists of route discovery and route maintenance models. The Dynamic MANET On-demand (DYMO) routing protocol enables reactive, multihop unicast routing between participating DYMO routers. The basic operations of the DYMO protocol are route discovery and route maintenance.

\section{DYMO}

Dynamic Manet on demand routing protocol is the fast routing protocol. It discovers the uncast routes among DYMO routers within the network in on demand fashion, offering improved converge in dynamic topology.

\section{1) Route discovery:}

When a source needs to send a data packet, it sends an RREQ to discover the route to the particular destination. After issuing an RREQ the or gin DYMO router waits for a route to be discovered. If the route is not obtained it si Waited and waiting time is calculated .Data Packets awaiting a route should be buffered by the source router . This buffer should have a fixed limited size. 


\section{2) Route maintenance}

It consists of two operations. In order to preserve routes in use, DYMO routers extend route lifetimes upon successfully forwarding a packet. In order to react to changes in the network topology, DYMO routers monitor links over which traffic is flowing.

When a data packet is received for forwarding and a route for the destination is not known or the route is broken, then the DYMO router of source of the packet is notified. A Route Error (RERR) is sent toward the packet source to indicate the current route to a particular destination is invalid or missing. When the source's DYMO router receives the RERR, it deletes the route. If the source's DYMO router later receives a packet for forwarding to the same destination, it will need to perform route discovery again for that destination. DYMO uses sequence numbers to ensure loop freedom [Perkins99]. Sequence numbers enable DYMO routers to determine the order of DYMO route discovery messages, thereby avoiding use of stale routing information.

Comparison between on demand routing protocols

\begin{tabular}{|c|c|c|c|}
\hline $\begin{array}{c}\text { SOURCE } \\
\text { ROUTE }\end{array}$ & DSR & AODV & DYMO \\
\hline $\begin{array}{c}\text { NEIGHBOUR } \\
\text { DIRECTION }\end{array}$ & NO & $\begin{array}{c}\text { HELLO } \\
\text { messages }\end{array}$ & Messages will come \\
\hline Route storage & Route cache & Route table & Route Table \\
\hline $\begin{array}{c}\text { Loop freedom } \\
\text { maintenance }\end{array}$ & Source router & $\begin{array}{c}\text { Sequence } \\
\text { number }\end{array}$ & Sequence number \\
\hline $\begin{array}{c}\text { Multipath } \\
\text { Raintenance }\end{array}$ & $\begin{array}{c}\text { Yend rerr } \\
\text { packet } \\
\text { Salvage } \\
\text { choose } \\
\text { another route }\end{array}$ & $\begin{array}{c}\text { Send RERR } \\
\text { route }\end{array}$ & $\begin{array}{c}\text { Sediscovery } \\
\text { Re route discovery }\end{array}$ \\
\hline $\begin{array}{c}\text { Multicast } \\
\text { capability }\end{array}$ & No & Yes & Yes \\
\hline
\end{tabular}

\section{SIMULATION SETUP}

The qualnet 5.0 network simulator is used for analysis. In the scenario the ftp connection is used as a MAC layer, Here we study the performance of dynamic nature of communication network. Simulation is wireless and FTP algorithms are used. in this scenario we sue $1500 \mathrm{~m} * 1500 \mathrm{~m}$ orgin.The mobility model used here is random waypoint in the rectangular field. The multiple nodes are applied and destination nodes are attached.

Simulation parameter

\begin{tabular}{|c|c|}
\hline parameter & Value \\
\hline Area & $1500 \mathrm{~m}^{*} 1500 \mathrm{~m}$ \\
\hline Simulation Time & $91,120,200 \mathrm{sec}$ \\
\hline Channel frequency & $2.4 \mathrm{ghz}$ \\
\hline Data rate & $2 \mathrm{Mhz}$ \\
\hline Path loss model & Two way model \\
\hline Mobility model & Random way point \\
\hline Packet size & 512 bytes \\
\hline Mac protocol & Ieee 802.1 \\
\hline
\end{tabular}

VI. MOBILITY MODEL:

Random way point is used which means a message is sent from source to destination by step by step and it have little delay in each node and then it travel from one node to other node. 


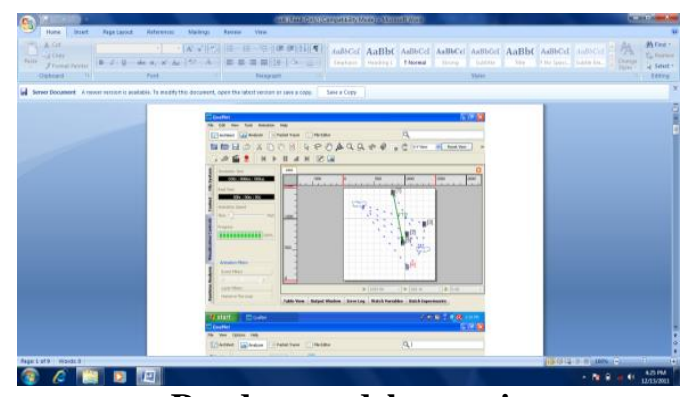

Random model scenerio

\section{PERFORMANCE METRICS}

The following four important performance metrics are considered for evaluation of these on demand routing parameter

Packet delivery fraction: The ratio of the data packets delivered to the destination to those generated by the CBR sources

Average end to end delay of data packets: This includes all possible delays caused by buffering during route discovery latency,queing at the interface queue retransmission delay rate at the MAC ,end propagation

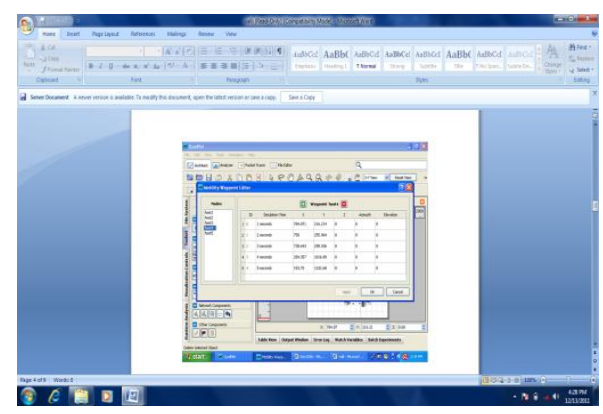

Throughput: it is average rate of successful data packets received at destination. It is usually measured by bits per second

End to end delay: A special packet is sent from source and destination and calculates the difference between the send time and receive time.
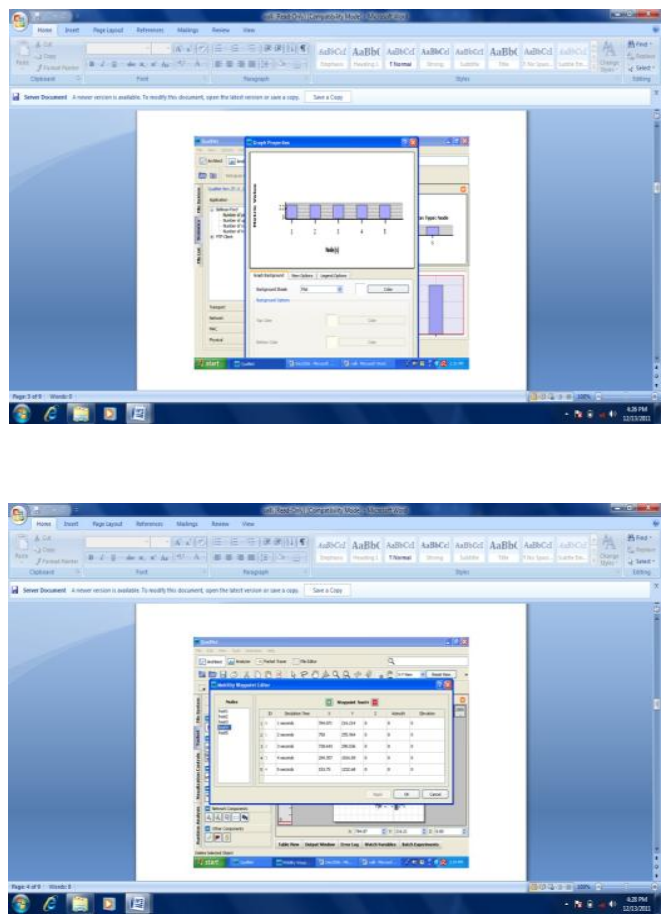


\section{CONCLUSION}

This paper provides explanation anlaysis of ondemand routing protocols DYMO using qualnet5.0. It has also performed a comparision among the performance metrits like pause time,throughput,jitter and end to end delay.There are various simulators for measuring the protoocls , here we use the new version of qualnet5.o for Dymo protocol. Graphs and simulation can show the performance the DYMO enhanced version of AODV . Dymo will perform better than some protocols. The effect of increasing nodes was also analyzed easily by the qualnet5.o.Hence the performance of the DYMO is better than ADOV andLDSR.From the result of our studies and analysis it can be concluded that, on an average DYMO Iis better than AODV and DSR. The work done in this research aims to develop an understanding of the effects of mobility patterns on routing performance. In future, we intend to study mobility models to determine the MANET protocol best suited to military mobile adhoc networks.

\section{REFERENCES:}

[1] Scalable Network Technology, "QualNet5.0 simulator "tutorial and QualNet um,http://www.scalablenetworks.com/forums

[2] Aisha Hassan Abdulla, A Simulation Based Performance Analysis of Reactive Routing Protocols in Wireless Mesh Networks, 2009 IEEE DOI 10.1109/ICFN.2009

[3] PERKINS, C,. BELDING ROYER, E, S. Das: Ad hoc On Demand Distance Vector (AODV) Routing, frp://ftp.isi.edu/innotes/rfc3561.txt

[4] Stefano. B,. et. al., "Mobile ad hoc Networking", IEEEPress, July 2004.

[4] Chakeres I, et. al., "Dynamic MANET On-demand (DYMO) Routing", IETF, June 2006

[5] Marco Fiore code - Webpage, http://www.tlcnetworks. polito.it/fiore/,Accessed on September 2007

[6] Arun Kumar B. R., Lokanatha C. Reddy, Prakash S. Hiremath "Performance Comparison of Wireless Mobile Ad-Hoc Network Routing Protocols" IJCSNS International Journal of Computer Science and Network Security, VOL.8 No.6, pp. 3

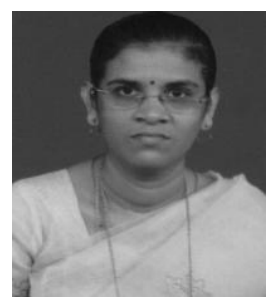

C.Chandralekha is from Coimbatore. She has completed her M.C.A. Degree at Madurai Kamaraj University campus, Madurai. She was working as Lecturer at St.Joseph's college of Engineering ,Jeppiaar Nagar, Chennai. Currently She is a Research scholar and Associate Professor at SNS College of Technology, Coimbatore. Her area of research is MANET

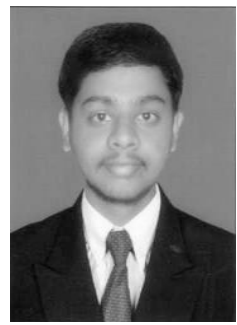

R.Balachandar is from Coimbatore born in October 16, 1990.He has completed his Under Graduation B.Sc. Degree in Computer Science from Bharathiyar University, Coimbatore. He is currently pursuing MCA degree from Anna University, Chennai. He is a Microsoft Certified IT Professional ; Oracle Certified Java Professional. His areas of interest include Network Security, Mobile computing. 